
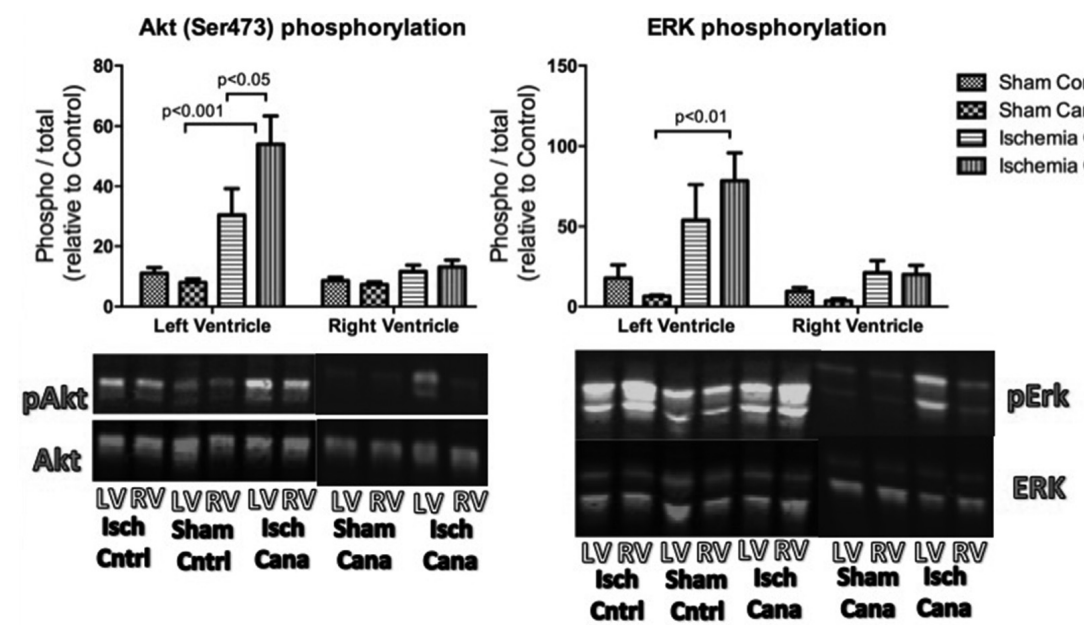

Abstract BS10 Figure 2

ketone and glucose did not seem to contribute to the observed cardioprotection. Further studies are vital to identify the hormone responsible for activating pro-survival pathways but our data highlights the potential use of SGLT2 inhibitors as a novel cardioprotective therapy in high-risk cardiovascular patients regardless of diabetic status.

Conflict of interest None

\section{BS11 ABSTRACT WITHDRAWN}

\section{BS12 INVESTIGATING OPTIMAL PLATELET PREPARTIONS TO PROMOTE ANGIOGENESIS AND WOUND HEALING}

Sarah Jones, Amelia Drysdale*, Fahmina Khanom, Sadiyah Kalsoom, Anuoluwapo Opadiran, Ryan Riley. Manchester Metropolitan University

\subsection{6/heartjnl-2019-BCS.175}

Background Platelets are a rich source of growth factors and play an important role in wound healing following vascular injury. Due to this tissue regenerative capacity the use of platelet rich plasma (PRP) to promote wound healing has gained traction over recent years. Despite increased use of PRP as a regenerative therapy, the optimal PRP preparations have not been investigated and the platelet-derived growth factors, which promote wound healing, have not been fully characterised. The aim of this study was to investigate the wound healing capacity of different PRP preparations and identify the growth factors released.

Methods Blood was obtained from healthy volunteers $(n=5)$ and PRP and platelet poor plasma (PPP) prepared by centrifugation. Plasma preparations were stimulated with thrombin (1Uml), or ADP $(10 \mu \mathrm{M})$ for $3 \mathrm{~min}$ and platelets removed by centrifugation. The different plasma preparations containing platelet releasate were used as treatments in HUVEC scratch assays and angiogenesis tube formation assays $(n=5)$. Plasma preparations from all five donors were pooled and a proteome profiler assay used to determine the angiogenic modulators released from platelets by thrombin and ADP.
Results Thrombin treated PRP and PPP significantly enhanced HUVEC wound closure compared to PPP alone, $(\mathrm{P}<0.02)$. ADP also significantly enhanced PPP but not PRP induced wound healing $(\mathrm{P}<0.008)$. The wound closure stimulated by thrombin was significantly greater than that observed with ADP $(\mathrm{P}<0.005)$. The presence of platelet releasate from PRP had no significant effect on the extent of wound closure compared to ADP/PPP or thrombin/PPP, suggesting that enhanced wound healing was through direct effects of thrombin and ADP on HUVECs. In contrast, the angiogenesis assay demonstrated that platelet releasate stimulated with ADP and thrombin increased average tube length, 16-fold and 6-fold respectively when compared to agonist stimulated PPP. Reducing the number of platelets in PRP by $50 \%$ markedly reduced thrombin/PRP evoked tube formation, supporting the notion that it was the releasate positively regulating angiogenesis. Analysis of the platelet releasate stimulated by both agonists demonstrated a number of common pro- and antiangiogenic proteins including TGF- $\beta$, PDGF, PIGF, FGF, thrombospondin 2 and vasohibin. Differential release was observed for a number of antiangiogenic proteins, including serpin B5, serpin F1 and angiostatin, which were only released by ADP. In contrast, the proangiogenic growth factor angiopoietin-1, was only released by thrombin. Interestingly some angiogenic modulators found in PPP, were markedly reduced by thrombin releasate, indicating protein degradation by either thrombin or enzymes contained within the releasate. Many of the proteins reduced by thrombin releasate were antiangiogenic suggesting that thrombin may induce tube formation by removing negative regulators in addition to releasing growth factors, which positively regulate angiogenesis.

Conclusion Platelets contain a large number of proteins, which can modulate wound healing and angiogenesis, and therefore have the potential to be used therapeutically to treat chronic wounds. Different agonists however differentially release the pro- and antiangiogenic growth factors stored in platelet granules. Further work is therefore required to identify the optimal PRP preparation to promote wound healing.

Conflict of interest none 